\title{
Human amniotic mesenchymal stem cells to promote/suppress cancer: two sides of the same coin
}

\author{
Ameneh Jafari ${ }^{1,2^{*}}$ (D), Mostafa Rezaei-Tavirani ${ }^{2}$, Behrouz Farhadihosseinabadi ${ }^{3}$, Hakimeh Zali ${ }^{4}$ and Hassan Niknejad ${ }^{5^{*}}$
}

\begin{abstract}
Cancer is a leading cause of death in both developed and developing countries, and because of population growth and aging, it is a growing medical burden worldwide. With robust development in medicine, the use of stem cells has opened new treatment modalities in cancer therapy. In adult stem cells, mesenchymal stem cells (MSCs) are showing rising promise in cancer treatment due to their unique properties. Among different sources of MSCs, human amniotic fluid/membrane is an attractive and suitable reservoir. There are conflicting opinions about the role of human amniotic membrane/fluid mesenchymal stem cells (hAMSCS/hAFMSCs) in cancer, as some studies demonstrating the anticancer effects of these cells and others suggesting their progressive effects on cancer. This review focuses on recent findings about the role of hAMSCs/hAFMSCs in cancer treatment and summarizes the suppressing as well as promoting effects of these cells on cancer progression and underling mechanisms.
\end{abstract}

Keywords: Amniotic mesenchymal stem cell, Tumor suppressing, Tumor promoting, Angiogenesis, Apoptosis

\section{Introduction}

Cancer is one of the leading causes of mortality worldwide [1]. Over the past decades, numerous studies have been conducted to find new therapeutic approaches with greater effectiveness and fewer side effects to replace conventional therapies [2]. Despite advancing progresses in the cancer survival rate, many conventional treatments need to be replaced by new and innovative ones. In this light, great potential of stem cells underscores their therapeutic index in treatment of cancers. In particular, numerous experimental studies have highlighted the mesenchymal stem cells (MSCs) as promising tool in cancer therapies [3-5].

The discovery of mesenchymal stem cells (MSC), as the non-hematopoietic stem cells, goes back to1960s

\footnotetext{
*Correspondence: amenehjafari@gmail.com; niknejad@sbmu.ac.ir 'Department of Basic Sciences, Faculty of Paramedical Sciences, Shahid Beheshti University of Medical Sciences, Tehran, Iran

${ }^{5}$ Department of Pharmacology, School of Medicine, Shahid Beheshti University of Medical Sciences, Tehran, Iran

Full list of author information is available at the end of the article
}

when Friedenstein and colleagues isolated these cells from the bone marrow [6]. MSCs are naturally developed in the human body and can be easily obtained from a number of adult and fetal tissues such as the heart, liver, kidney, skeletal muscle, adipose and connective tissue, synovial tissue, placenta, and umbilical cord [7]. These cells express some specific cell markers like CD73, CD90, and, CD105; however, they do not express CD34, CD45, CD14, CD11b, CD79-a, CD19, and HLADR [8]. MSCs are known as multipotent cells with a great potential of self-renewal and differentiation into a versatile cell linage such as adipocytes, chondrocytes, myocytes, osteocytes, fibroblasts, neurons, and epithelial cells $[9,10]$. In general, MSCs show intrinsic antitumor property due to their anti-proliferative activity, induction of apoptosis, and suppression of angiogenesis [11]. However, it is not fully understood how these cells govern their anti-cancer effects. Moreover, the anti-cancer effect of MSCs seems to be cancer type-related. For example, bone marrow-derived MSCs stimulated osteosarcoma, 
breast cancer, colorectal tumor, and gastric cancer, while those isolated from the adipose tissue or umbilical cord showed inhibitory effect on a group of cancer types including lung, liver, glioma, and prostate cancers. The rationale behind this discrepancy remains unclear; however, some reasons have been proposed such as lack of a standard experimental method, challenges with isolation of MSC subgroups due to the lack of specific markers, and unpredictable behaviors of these cells resulting from the strong influences of microenvironment surrounding them [12].

To date, most preclinical studies have focused on bone marrow-derived MSCs, although these cells might not be the best available source. The bone marrow-derived MSCs have been used in various clinical therapies to treat several cancer types including glioma, ovarian, and prostate cancers [13]. However, using these cells faces with some challenges including (i) need for aggressive operations to harvest them from the bone marrow (ii) limited number of the isolated cells (iii) and decreased ability to differentiate as the patient ages [14]. There are other accessory sources for harvesting MSCs such as the adipose tissue, umbilical cord, and Wharton's jelly [15, 16]. Adipose derived-MSCs are highly suitable alternative due to ease of tissue collection with higher number and favorable proliferation capacity in vitro compared with the other sources [17]. MSCs derived from the umbilical cord blood and Wharton's jelly are also isolated through a completely non-invasive and simple procedure $[18,19]$. In recent years, the MSCs derived from the amniotic fluid and amniotic membrane have been introduced as an attractive and potent stem cell sources for clinical application because of their easy, safe, and painless collection procedures with minimized ethical issues. In recent years, placenta-derived MSCs, especially those isolated from human amniotic membrane (hAMSCs) and amniotic fluid (hAFMSCs), are more popular due to their non-invasive isolation techniques, large-scale supply, genome stability, non-tumorigenic, being able to interact with different tissue environments, and acceptable ethical issue [20,21].

Until now, various studies have been conducted to evaluate the effect of hAMSCs/hAFMSCs on cancer cell behaviors. Some studies have revealed that hAMSCs/ hAFMSCs induced tumor growth and metastasis [22]. However, reports confirming the anti-cancer effects of these cells are also available in literature [23, 24]. The controversial results and poor understanding of mechanisms behind the effect of hAMSCs/hAFMSCs on cancer cells hamper those researchers who are trying to use such cells in clinic [25]. This review article highlights recent findings regarding the effect of hAMSCs/hAFMSCs on cancer cells and complies evidences supporting both anticancer and cancer growth promotor effect of these cells.

\section{MSCs derived from the amniotic fluid and amniotic membrane}

Amniotic fluid (AF) fills the amnion cavity that surrounds the fetus and serves as the protective and provider fluid, ensuring the fetus to earn required protection and nutrients during embryogenesis $[26,27]$ (Fig. 1). The AF is mainly composed of water, chemical substances, and cells. In the early twentieth centuries, it is found that AF contains abundant stem cells [27]. Across various cellular subpopulations of $\mathrm{AF}$, amniotic fluid mesenchymal stem cells (AFMSCs) are particularly

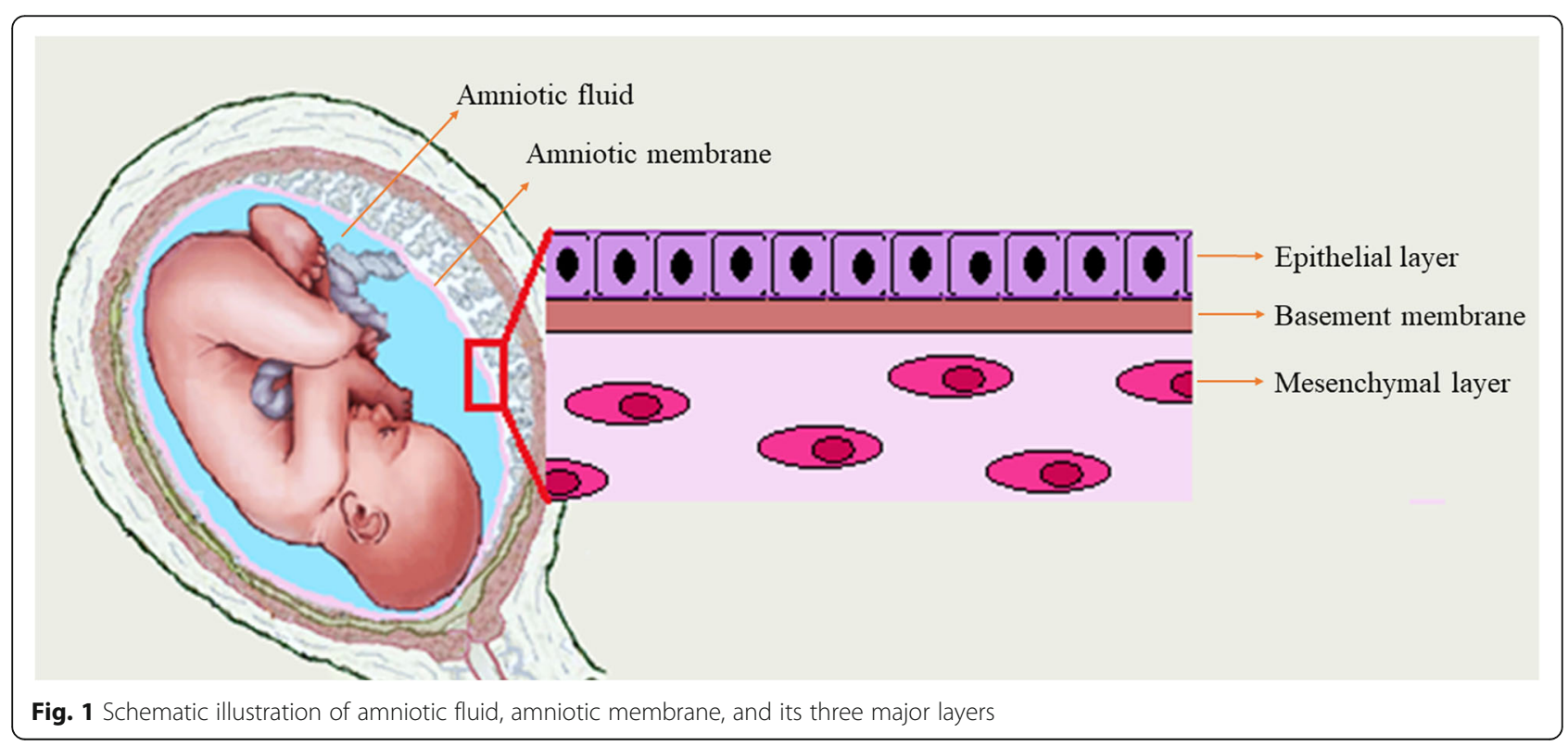


favorable because of their therapeutic potential. These cells can be easily collected by amniocentesis in the second and third trimesters or even at the end of pregnancy $[28,29]$. It not only provides a safe way to isolate these cells with a minimum risk for the mother and fetus, but also avoid any ethical issues that normally found in work with embryonic stem cells [24, 30]. These cells express the stem cell markers including Oct-4, c-Myc, Sox2, Nanog, and SSEA3 that well-confirm their pluripotency $[27,31,32]$. In addition to the pluripotency markers, AFMSCs exhibit high levels of several MSC markers including CD29, CD44, CD73, CD90, CD105, CXCR4, stromal cell-derived factor 1 receptor (SDF-1), CD146, CD166, and CD184 [32]. Regarding the pluripotent capacity of AFMSCs, they are able to differentiate into adipogenic, chondrogenic, and osteogenic lineages under a specific condition [33, 34].

Amnion or human amniotic membrane (hAM) is the innermost layer of fetal membranes, which is directly in contact with the amniotic fluid and the fetus. The hAM consists of three major layers include the epithelial layer, the basement membrane, and the mesenchymal layer [29, 35] (Fig. 1). Human amniotic mesenchymal stromal cells (hAMSCs) can be harvested from the mesenchymal layer of hAM by a simple enzymatic digestion procedure ended to isolate about $10^{7}$ cells from a single hAM $[36,37]$. Similarly to MSC, hAMSCs are pluripotent stem cells and able to differentiate into the three germ layers [38]. hAMSCs express specific surface markers such as CD14, CD29, CD44, CD49, CD90 (Thy-1), CD105 (endoglin), CD166, and SSEA-3/4 [39], while they do not express HLA-DR (MHC class II), CD19, CD45, and CD133 [32]. RT-PCR results also confirmed that these cells expressed stem cell markers like SSEA-4, Nanog, and OCT4 [40].

Stem cell properties of AMSCs/AFMSCs along with their low immunogenicity introduce them as a promising source for applications in the field of tissue engineering, regenerative medicine, and cell therapy [41, 42]. Some unique features of AMSCs/AFMSCs that make them the suitable candidates for therapeutic care diseases ranging from wound healing to cancer therapy are listed in Table $1[43,44]$.

\section{Tumor-suppressing effects of hAMSCs/hAFMSCs Proapoptotic effects of hAMSCs/hAFMSCs}

MSCs are strong proliferation inhibitors of immune cells like the natural killer cells (NK cells). The same results have been reported on numerous cancer types such as breast cancer [45], hepatoma [46], ovarian cancer [23], and glioma [47]. It is reported that MSCs exert their growth inhibitory effect through MSC-secreted prostaglandin E2 (PGE2), IL-6, indoleamine2,3- dioxygenase (IDO), TGF- $\beta 1$, and NO [48, 49]. Recently, Aziz et al. found a significant reduction of survival rate in the
Table 1 The unique characteristics of amniotic fluid/membrane mesenchymal stem cells $[43,44]$

\begin{tabular}{ll}
\hline Feature & Description \\
\hline Accessibility & No need to use invasive procedures for collection \\
Availability & $\begin{array}{l}\text { Readily available in large number for } \\
\text { transplantation }\end{array}$ \\
Plasticity & Ability to differentiate into different cell types \\
Mitotic stability & Karyotype stability during several cell divisions \\
Ethical & Use without ethical concerns. \\
acceptability & \\
Low-risk & No need to be concern regarding transplant-related \\
& diseases, such as immunoreaction and cancer \\
\hline
\end{tabular}

ovarian cancer cells after co-culturing with hAFSCs compared with the control group [23]. The authors reported a significant increase in the expression of P53 and P21 genes, and also, the genes involved in apoptosis in the treated cancer cells. In that study, the expression rates of cyclin D1 and B1 genes were lower than the control group, indicating blockage of cell cycle progression from phase G0 to G1 and inhibition of phase $S$ commencement [23]. hAMSCs are not only able to inhibit the proliferation of immune cells [50] but also they promote the apoptosis of $\mathrm{B}$ and $\mathrm{T}$ lymphocytes [51]. Kang et al. showed a significantly decrease in the growth and proliferation of cancer cells in vitro and in vivo. Through evaluating the cytokines involved in this phenomenon, they pointed out the key roles of hAMSCreleased factors such as TNF- $\alpha$, TGF- $\beta$, TNF- $\beta$, and IFN- $\gamma$ as the proapoptotic agents [52].

Aquaporins, which serve as channels involved in the transfer and control the water contents in cells [53], are also responsible for the anti-proliferative and proapoptotic activities of the AM cells [54]. Indeed, activation of peroxisome proliferator-activated receptor- $\gamma$ (PPAR- $\gamma$ ) signaling by AM ingredients resulted in the upregulation of aquaporins and consequently apoptosis in cancer cells $[55,56]$.

In a study, intravenous administration of amniotic mesenchymal cells in glioma C6 tumors in a single and multiple doses reduced the tumor size by approximately 30 and $50 \%$, respectively. High expression of caspase-3, caspase-8, and Bax, and low expression of Bcl-2 in tumor cells after treatment with amniotic mesenchymal cells confirmed the apoptosis as the main mechanism of cancer cell death. You et al. reported that injection of genetically modified hAFMSCs expressing IL-2 led to induction of apoptosis in the ovarian cancer nude mice models [57].

\section{Anti-angiogenesis effects of hAMSCs/hAFMSCs}

Given the necessity and importance of angiogenesis for tumor growth and metastasis, controlling of angiogenesis in tumors is an exciting strategy to inhibit cancer 
progression [58]. In contrast to some reports claiming the release of angiogenic factors by MSCs, Lee et al. observed that MSC-derived extracellular vehicles (EVs) inhibited angiogenesis in tumor cells through downregulation of VEGF [59]. MSC-derived exosomes contain a wide range of proteins and RNA, reflexing the nature of the cells producing them. Among these mediators, miR16 which is known for targeting VEGF, have been enriched in MSC-derived exosomes that partly explained the antiangiogenic effect of these vesicles [60].

In a paracrine manner, hAMSCs secrete mediators like thrombospondin, TIMP 1-4, pigment epitheliumderived factor (PEDF), collagen XVIII, and endostatin that contribute in suppressing cancer cell migration and cancer angiogenesis [61, 62]. Downregulation of factors such as VEGF, PDGF-AA, PDGF-BB, BMP-4, and CXCL16 has been reported after co-culturing of cancer cells with MSCs. These results have been related to the anti-angiogenesis and anti-migration effects of MSCs [63-65]. Meng et al. observed similar results following co-culture of SPC-A-1 cells with hAMSCs [66].

HIF1 $\alpha$, a client protein of HSP90, involves in activation of VEGF $[67,68]$. In addition, HIF1 $\alpha$ by activating MMP-2 induces invasion and metastasis of tumor cells [69]. The crucial role of HSP90 in stabilization and activation of HIF1 $\alpha$ has been proved. It seems that inhibition of HSP90 is one of the anticancer mechanisms of amniotic stem cells [70].

\section{Anti-inflammatory effects of hAMSCs/hAFMSCs}

Inflammation is a process involving the activation, recruitment, and function of innate and adaptive immune cells. This process plays critical roles in host protection against pathogens, tissue repair, regeneration, and remodeling, and it is essential for regulating tissue homeostasis [71]. Chronic inflammation prompts cancer development through different mechanisms. Indeed, tumors have usurped the pathways built to mediate immunity to infection and promote tissue homeostasis to their advantage [72]. In order to form an inflammatory tumor microenvironment, cancer cells, surrounding stromal and inflammatory cells, participate in a well-orchestrated reciprocal interaction [73]. It has been shown that hAMSCs have immunosuppressive activities because of their ability to secrete HLA-G, their low expression of MHC class I, and no expression of MHC class II [74-76]. These cells are able to reduce inflammatory response inhibit inflammatory cell infiltrate; reduce migration, recruitment, and activity of a broad range of immune cells; and suppress neutrophil extracellular traps $[76,77]$. In a study, hAMSCs were used in rabbit corneal alkali-burn models. The obtained result indicated that intracameral hAMSC injection could induce an anti-inflammatory and anti-fibrotic environment and subsequently stimulate corneal wound healing [76]. Based on evidence, the hAMSC and hAMSC-derived conditioned medium (hAMSC-CM) possess the ability to suppress $\mathrm{T}$ cell function, decrease the expression of Th1 and Th17 markers, increase T-regulatory (Treg) cells, and decrease NK cell cytotoxicity [78, 79]. Moreover, different studies have provided evidence of the antiproliferative effects of hAMSC-CM on lymphocytes and monocytes [77].

Studies have shown that the levels of proinflammatory cytokines are decreased by hAMSCs such as, tumor necrosis factor $\alpha$ (TNF- $\alpha$ ), interleukin (IL)-1 $\beta$, IL-6, IL-12, migration inhibitory factor (MIF), monocyte chemoattractant protein-1 (MCP-1), the soluble Fas-ligand (FAS-L), TNF-related apoptosis-inducing ligand (TRAI L), and IFN- $\gamma[77,80]$. In addition, it has been reported that hAMSCs are able to secrete or increase the production of immunosuppressive molecules such as hepatocyte growth factor (HGF), IL-10, TGF- $\beta$, and indoleamine 2,3 dioxygenase (IDO) [77, 81].

Considering the innate ability of MSCs to migrate toward the inflamed sites, recently, hAFMSCs have been used to deliver immunoregulatory cytokines [82, 83]. For example, Zhou and colleagues applied hAFMSCs as a delivering system to transfer IFN $\alpha$ to the cervical cancer tumor site. In that study, hAFMSCs were genetically modified to overexpress IFN $\alpha$ and then intravenously injected in order to migrate toward the tumor site in the mice model. Results showed the anticancer effects of IFN $\alpha$-AF-MSCs on the cervical tumors due to inhibition of angiogenesis, suppression of tumor cell proliferation, and induction of apoptosis in the tumor cells [84].

In general, it has been widely demonstrated that hAMSCs and their CM are capable of dampening in vitro inflammatory conditions by suppressing proliferation, inflammatory cytokine production, stimulatory, and cytotoxic activity of various subpopulations of immune cells, and inducing anti-inflammatory and regulatory functions of $\mathrm{T}$ cells and monocytes.

\section{Role of hAMSCs/hAFMSCs in cell cycle arrest}

The cell-cycle arrest is another anti-tumor mechanism of amniotic mesenchymal cells. Previous studies have indicated that MSCs isolated from different sources such as breast tissue, adipose tissue, and human palatine tonsils exert their antiproliferative effect by inducing cell-cycle arrest in the G0/G1 phase $[85,86]$. Based on microarray data, the antiproliferative effect of hAMSC is attributed to downregulation of cyclin D1, cyclin E1, cyclin $\mathrm{H}$, cyclin-dependent kinase (CDK) inhibitor $\mathrm{p} 15^{\mathrm{INK} 4 \mathrm{~b}}$, and CDK inhibitor $\mathrm{p} 21^{\mathrm{Waf1} / \mathrm{Cip} 1}$ as well as upregulation of retinoblastoma (RB). These events finally lead to G0/G1 cell cycle arrest in the cancer cells [87]. Riedel and colleagues suggested that human amniotic membrane-conditioned medium (hAM-CM) was able to inhibit DNA synthesis, cell viability, and cell cycle 
progression through decreasing Cyclin D1 and Ki-67 expression and increasing p21 and p53 expression [88]. The authors also observed upregulation of anti-oncomiRs such as miR-15a and miR-210 and downregulation of oncomiRs including miR-206 and miR-145 following treatment cancer cell lines (HepG2 and HuH-7 cells) with hAM-CM [88]. Several studies reported that hAMSCs could inhibit the positive regulators of the cell cycle such as proliferating cell nuclear antigen (PCNA) and the mini-chromosome maintenance complex (MCM2, MCM4, MCM5) [87, 89]. In addition, Cullin 1 (CUL 1 ), which mediates the degradation of various proteins including $\mathrm{p} 21$, is downregulated by amniotic mesenchymal stem cells [87].

\section{Tumor-promoting effects of hAMSCs/hAFMSCs}

In contrast to the anticancer effect of hAMSCs/AFMSCs that discussed above, various studies have shown that these cells promote tumor progression and metastasis by enhancing angiogenesis, upregulating Akt/mTOR signaling pathways, and promotion metastasis (Fig. 2) [90].

\section{Promoting angiogenesis effects of hAMSCs/hAFMSCs}

Tumor angiogenesis and neovascularization are important multistep processes that occur during tumor progression and metastasis [91]. MSCs due to their potential to secrete a wide variety of growth factors, chemokines, and cytokines including VEGF, bFGF, TGF- $\beta$, MCP-1, SDF1, angiopoietin, MMPs, CXCL2, CXCL8 monocyte chemoattractant protein, IL-6, IL-8, and placental growth factor can effectively induce angiogenesis [92-94]. In this context, hAMSCs are able to promote formation, stabilization, and maturation of new vessels [91, 95]. These cells could regulate vascular network remodeling through the release of angiogenic factors or differentiation into endothelial cells (Fig. 2) [61, 96]. Studies elucidated that hAMSCs contain some angiogenic factors including VEGF, bFGF, IL-6, IL-8, MIF, growth-related oncogene (GRO), monocyte chemoattractant protein-1 (MCP-1), and intravascular adhesion molecule (ICAM). One of the most potent proangiogenic factors is VEGF that is secreted by macrophages and keratinocytes and has a crucial role in promoting proliferation of vascular endothelial cells [97]. bFGF is another strong angiogenic factor that stimulates the proliferation and migration of vascular endothelial cells [98]. Furthermore, it is shown that hAMSCs increase the expression of MMP-1 and reduce the ratio of TIMP-1/ MMP-1 [99], which participates in tumor neovascularization, and subsequent metastasis [100]. In addition, hAMSCs can trigger various cell-signaling pathways needed for cell viability and neovascularization [96]. For example, hAMSCs promote angiogenesis by inducing

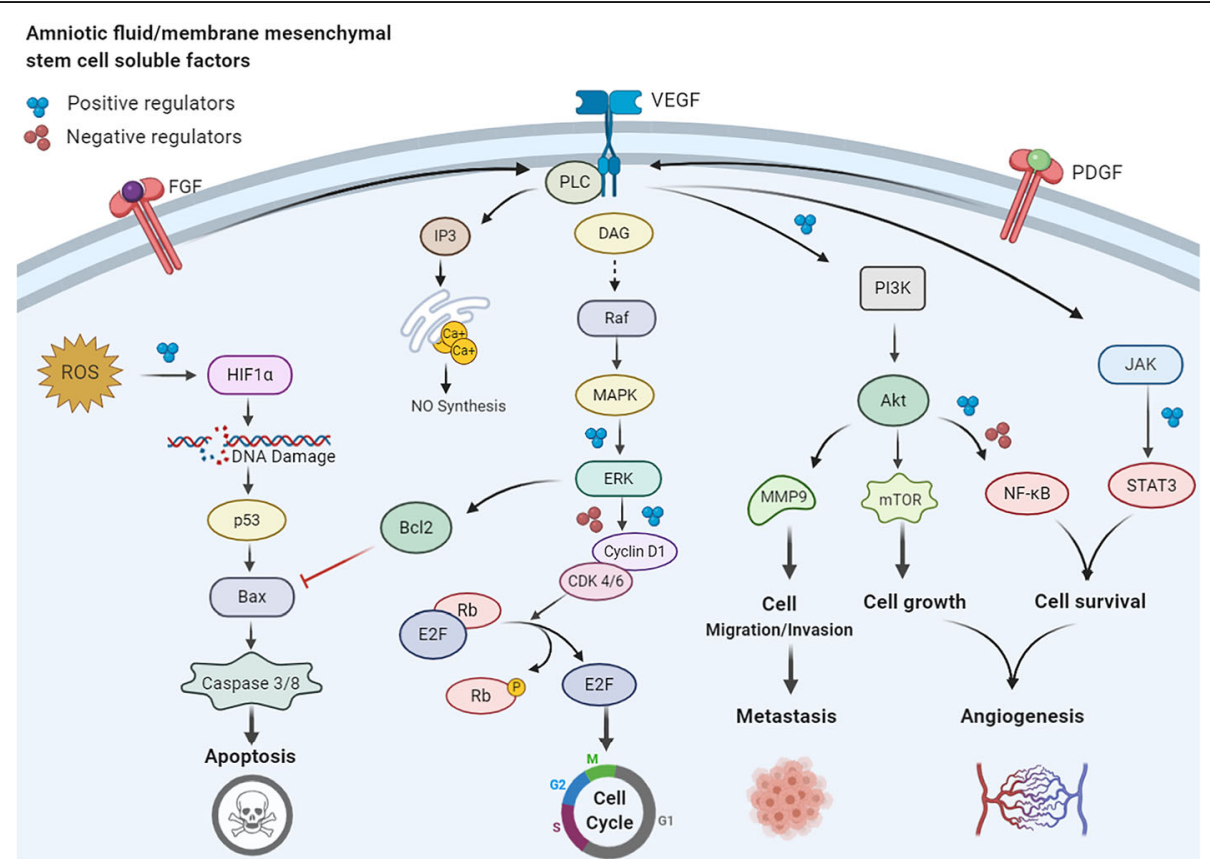

Fig. 2 The effect of regulators secreted by hAMSCs/hAFMSCs on various cancer signaling pathways. hAMSCs/hAFMSCs produce a wide verity of mediators that affect different signaling pathways in cancer cells. As two distinct cell fate, cell proliferation and apoptosis pathways are the most important sites of action of these mediators. Proteins such as VEGF and bFGF promote angiogenesis in cancer cells, helping them to supply nutrients and scape from the tumor microenvironment. In the opposite side, the mediators secreted from these cells through activation of different signaling pathways such as PI3K/AKT signaling lead the cancer cell to apoptosis 
the MAPK $1 / 2$ signaling pathway. Subsequently, the upregulation of phosphorylated ERK1/2 and RUNX2 are involved in the underlying mechanism [101]. Interestingly, hAMSC-CM could also induce neovascularization. $\mathrm{Wu}$ et al. found that hAMSC-CM promoted cell proliferation and angiogenesis in human aortic endothelial cells via secreted significant amounts of angiogeneticrelated growth factors including VEGF, EGF, HGF, IGF1, Ang-1, and bFGF [102]. Moreover, newly formed vascular network has been observed following treatment of human umbilical vein endothelial cells (HUVECs) with hAMSCs-CM [103]. Jeong et al. developed a gene modified hAMSC cell population overexpressing GCP-2 and SDF- $1 \alpha$ to evaluate its angiogenic potency. The Matrigel assay revealed the high angiogenic potential of the CM obtained from the genetically engineered cells to form new vessels from HUVECs [104].

AFSCs secrete EVs containing surface mesenchymal/ exosomal markers as well as angiogenic receptors such as VEGFRs. Salomon et al. revealed that EVs derived from placental MSCs promote microvascular endothelial cell migration and vascularization under hypoxic circumstances [105]. Recently, it is indicated that AFSCderived EVs carry specific mRNA and miRNAs which are involved in different biological functions [106, 107]. Sedrakyan and colleagues found that these EVs contain modulator miRNAs including those regulate VEGF levels (miR-16.1, miR-93), VEGF receptors (miR-16.1), and positive/negative regulators of the VEGF signal transduction cascade (miR-23a, miR-27a, miR-145, miR221, and miR-322) [108]. Moreover, the presence of a wide variety of markers on the surface or within the AFSC-derived EVs such as NOS, TGF- $\beta$, MAPK, PPAR, and Ang II probably regulate other important mechanisms involved in angiogenesis [109].

\section{Anti-apoptotic activity of hAMSCs/hAFMSCs}

Emerging evidence suggests that tumor progression occurs in adaptation with anaerobic conditions. Indeed, chronic inflammatory status, acidic $\mathrm{pH}$ conditions, nutrient deprivation, and hypoxia have been observed during tumor growth [110, 111]. Under stress conditions, MSCs can maintain their characteristics and functions such as differentiation potentials and self-renewal capacity by activating autophagy pathways. Zhang et al. observed that MSC-derived exosomes could able to promote proliferation and inhibit apoptosis of skin cells after heat stress in vitro [112]. As mentioned before, MSCs are capable to release a number of pro-survival and anti-apoptotic factors such as VEGF, IGF-1/2, bFGF, PDGF, SDF-1- $\alpha$, HGF, stanniocalcin-1 (STC-1), and NO [135-140]. Among them, VEGF and bFGF apply their anti-apoptotic effects by enhancing BCL-2 expression and increasing the BCL-2/Bax ratio in tumor cells [113]. On the other hand, the upregulation of PDGF and TGF- $\beta$ are usually associated with increased expression of VEGF and bFGF in cancer cells [114]. MSCs also produce IL-6 that causes tumor cells to resistance to chemotherapy through upregulation of BCL-2 and activation of STAT-3 [113, 115]. STC-1 and $\mathrm{NO}$ (in low concentration) can inhibit apoptosis in tumor cells and promote tumor cell survival [113].

Anna and colleagues demonstrated that MSCs isolated from the umbilical cord (HUCMSCs) enhanced normal proliferation and migration of skin fibroblasts and promoted wound healing in the murine model through paracrine signaling [116]. Recently, Li et al. reported that in vivo transplantation of hAMSCs and hAMSC-CM considerably improved re-epithelialization and wound healing through enhancing proliferation and inhibiting apoptosis of heat-injured skin cells. The authors speculated that activation of PI3K/AKT and GSK3 $\beta / \beta$-catenin signaling pathways might be responsible for the therapeutic impacts of hAMSCs/hAMSC-CM on the damaged skin cells [40]. Numerous studies have shown that hAMSCs contribute in activation of PI3K/AKT cell survival pathway by secreting different cytokines such as PAI-1, C-GSF, IL-6, IL-8, IL-11, OPN, HGF, TGFb1, RBP4, ANG-1, ANG-2, FAP, Galectin-1, DcR3, Follistatin, IGF-2, and MCP-1 [117]. Periostin, one of the matricellular proteins, is capable to activate the PI3K/AKT signaling pathway in tumor cells by interacting with integrin molecules [40]. This protein also can increase the migration and proliferation of epithelial cells and dermal fibroblasts via upregulating AKT/mTOR signaling pathway $[118,119]$. Cetinkaya et al. observed the decreased expression of p57 cell cycle inhibitory protein and PARP-1 apoptosis marker as well as increased expression of Ki67 and PCNA following hAMSC administration [120].

Tissue inhibitor of metalloproteinases-1 (TIMP-1) is a protein released by hAMSCs, showing proliferative and anti-apoptotic features [121]. In a renal carcinoma model, it was shown that EVs released from hWJ-MSCs triggered the growth and invasiveness of renal carcinoma cells (RCC) in a xenograft model of BALB/c nu/nu mice [122]. Recently, it is reported that hUCMSC-EVs contain a large amount of miR-410 that strongly stimulate the growth of lung adenocarcinoma cancer cells in a xenograft tumor model [123]. According to all the previous studies, it seems that hUCMC-EVs are involved in growth and progression of tumor cells. Therefore, the use of them as a carrier for oncology therapy (engineered hUCMSCEVs) may be a promising therapeutic strategy.

\section{Role of hAMSCs/hAFMSCs in promoting metastasis}

Epithelial-mesenchymal transition (EMT) is a biological phenomenon in which epithelial cells acquire mesenchymal cell features. It plays a crucial role in cancer progression including invasiveness, metastasis, and the 


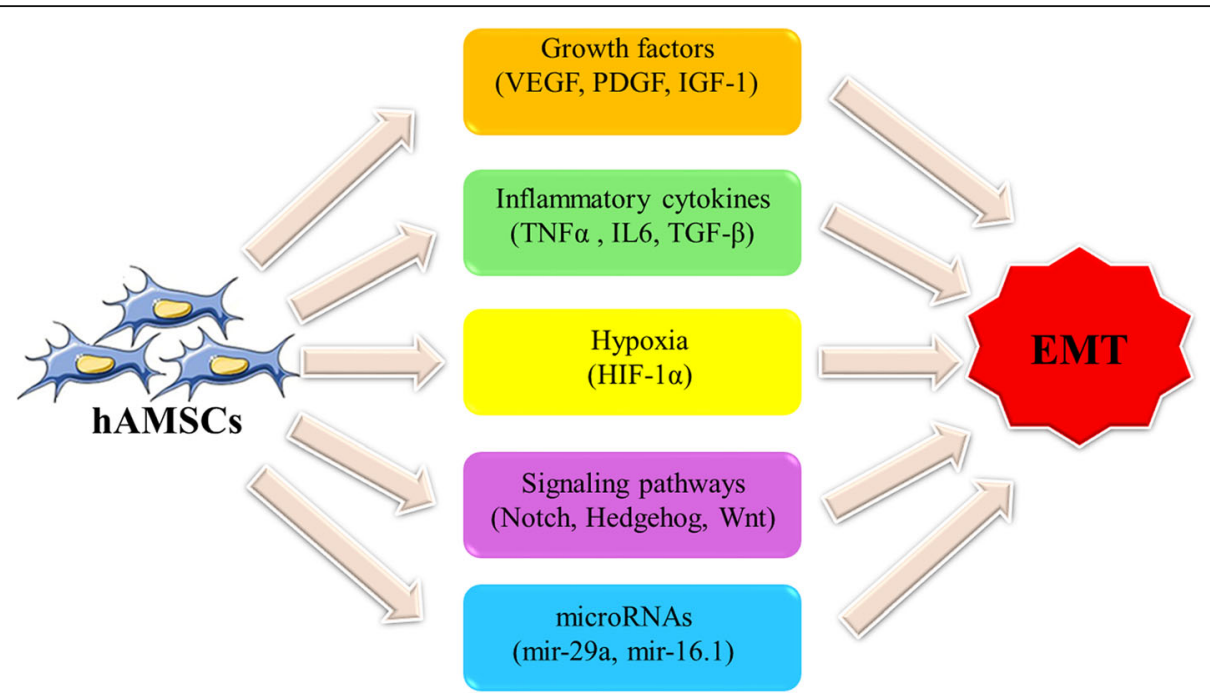

Fig. 3 The role various effectors secreted by hAMSCs in induction of EMT. The mediators that promote EMT in epithelial cells include a wide list ranging from proteins to non-coding RNAs

acquisition of resistance to apoptosis and chemotherapy [124-126]. There is an intertwined network of biological signaling pathways involved in the EMT process including Notch, Hedgehog, TGF- $\beta /$ Smad, NF- $\kappa B$, Wnt, and STAT3 pathways $[127,128]$. Moreover, the EMT process can be facilitated by the presence of reactive oxygen species (ROS) and inflammatory mediators [129]. More recently, it has been indicated that cross-talk between MSCs and tumor cells increases the metastatic potential and EMT in these cells $[130,131]$. Accordingly, hAMSCs by secreting a variety of paracrine factors such as FGF, EGF, VEGF, and, PDGF can significantly enhance or promote the EMT process (Fig. 3) [132]. In addition, studies have verified the presence of various types of MVs secreted by hAMSCs that take a role in this context [133]. It is found that these vesicles and their cargos play a key role in downregulation of
MMP-1, MMP-13, TNF- $\alpha$, IL-6, IL-1 $\beta$ genes, and the restoration of TGF- $\beta$ expression $[133,134]$.

\section{Conclusion}

Human amniotic mesenchymal stem cells act as a double-edged sword where they show either anti or protumorigenic effects (Fig. 4).

hAMSCs/hAFMSCs appear to be tumor promoter because these cells stimulate cancer cells' growth, proliferation, invasion, metastasis, and tumor angiogenesis. On the other hand, there are many reports confirming the tumor suppressor effect of hAMSCs/hAFMSCs. Given that these cells exhibit unique features including stable characteristics, low immunogenicity, and nontumorigenicity, they are an attractive candidate to use in the clinic. However, conflicting results about the effect

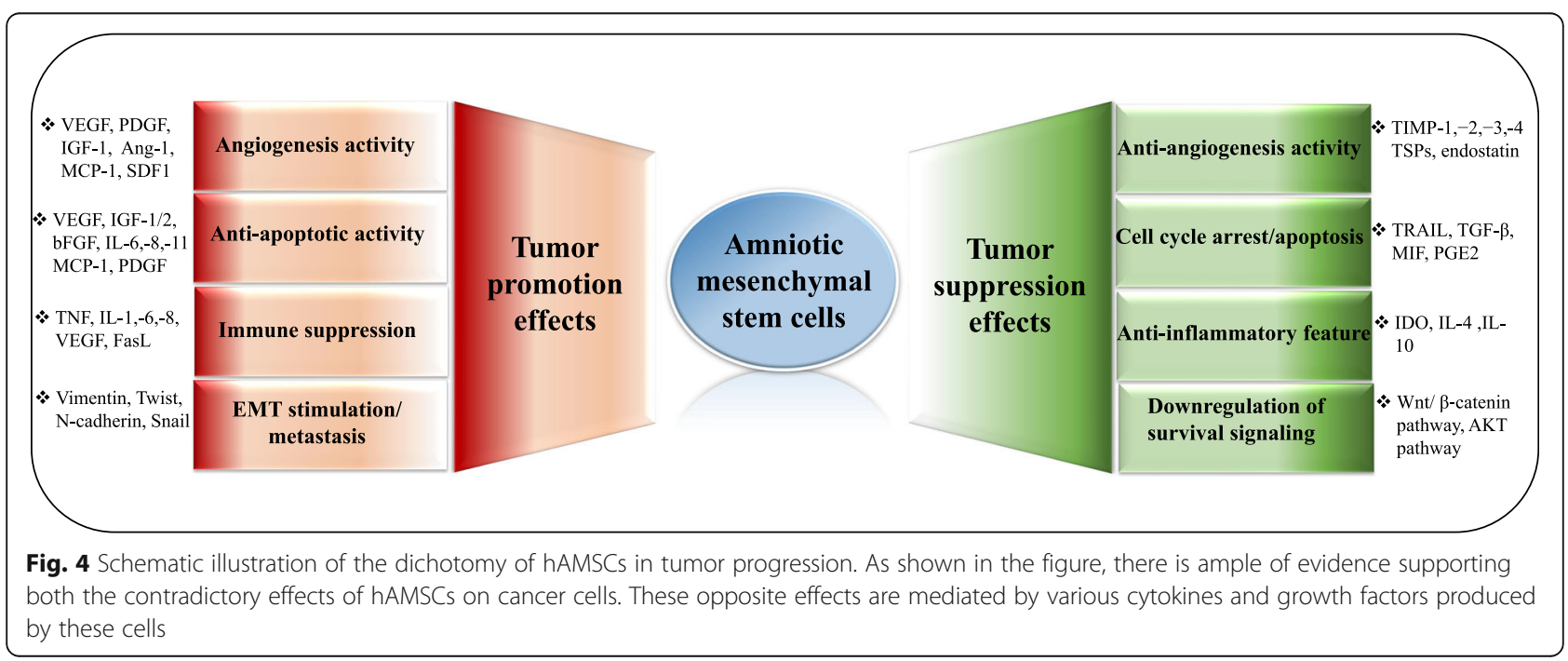


of these cells on tumors have cast doubt on their clinical applications.

Despite unpredictable behaviors of these cells in response to different cancer types, researchers can benefit from genetically modified hAMSCs/hAFSCs to bold anticancer side of these cells in cancer therapy. These genetically modified MSCs could be developed by two main strategies including overexpression of antitumorigenic mediators (interferons, interleukins, chemokines, and proapoptotic molecules) and encoding prodrugs or gene-directed enzymes in these cells. Moreover, hAMSC/hAFSC-derived exosomes can be used directly or as a carrier for delivering different anticancer drugs for treatment of different tumors.

\section{Abbreviations}

CM: Conditioned medium; CTL: Cytotoxic T lymphocyte; CXCR4: Chemokine (C-X-C motif) Receptor type 4; IDO: Indoleamine 2,3 dioxygenase; HGF: Hepatocyte growth factor; HIFs: Hypoxia-inducible factors; hAMSCs: Human amniotic MSCs; hAFMSCs: Human amniotic fluid MSCs; hUCMSCs: Human umbilical cord MSCs; hUCMSC-EVs: hUCMSC-derived extracellular vesicles; PI3K: Phosphoinositide 3-kinase; TRAIL: TNF-related apoptosis-inducing ligand; MSCs: Mesenchymal stem cells; NO: Nitric oxide; SDF1: Stromal cell-derived factor-1; TGF- $\beta$ : Transforming growth factor-beta; TNF-a: Tumor necrosis factor-alpha

\section{Acknowledgements}

This study supported by the School of Medicine, Shahid Beheshti University of Medical Sciences (NO. 16785)

\section{Authors' contributions}

AJ and $\mathrm{HZ}$ contributed to the conceptualization. MRT and $\mathrm{HN}$ supervised the study. AJ contributed to the investigation and wrote the original draft of the manuscript. BF contributed to finalizing the review. The authors read and approved the final manuscript.

\section{Funding}

Not applicable.

\section{Availability of data and materials}

Not applicable.

Ethics approval and consent to participate

Not applicable.

\section{Consent for publication}

Not applicable.

\section{Competing interests}

The authors declare that they have no competing interests.

\section{Author details}

${ }^{1}$ Department of Basic Sciences, Faculty of Paramedical Sciences, Shahid Beheshti University of Medical Sciences, Tehran, Iran. ${ }^{2}$ Proteomics Research Center, Faculty of Paramedical Sciences, Shahid Beheshti University of Medical Sciences, Tehran, Iran. ${ }^{3}$ Hematopoietic Stem Cell Research Center, Shahid Beheshti University of Medical Sciences, Tehran, Iran. ${ }^{4}$ Department of Tissue Engineering and Applied Cell Sciences, School of Advanced Technologies in Medicine, Shahid Beheshti University of Medical Sciences, Tehran, Iran. ${ }^{5}$ Department of Pharmacology, School of Medicine, Shahid Beheshti University of Medical Sciences, Tehran, Iran.
Received: 17 August 2020 Accepted: 27 January 2021

Published online: 12 February 2021

\section{References}

1. Jafari AR-TM, Salimi M, Tavakkol R, Jafari Z. Oncological emergencies from pathophysiology and diagnosis to treatment: a narrative review. Soc Work Public Health. 2020;35(8):689-709.

2. Pucci C, Martinelli C, Ciofani G. Innovative approaches for cancer treatment: current perspectives and new challenges. ecancer Med Sci. 2019:13:961-87.

3. Tan L, Dai T, Liu D, Chen Z, Wu L, Gao L, et al. Contribution of dermalderived mesenchymal cells during liver repair in two different experimental models. Sci Rep. 2016;6:25314.

4. Maria AT, Toupet K, Maumus M, Fonteneau G, Le Quellec A, Jorgensen C, et al. Human adipose mesenchymal stem cells as potent anti-fibrosis therapy for systemic sclerosis. J Autoimmun. 2016;70:31-9.

5. Cavarretta IT, Altanerova V, Matuskova M, Kucerova L, Culig Z, Altaner C. Adipose tissue-derived mesenchymal stem cells expressing prodrugconverting enzyme inhibit human prostate tumor growth. Mol Ther. 2010; 18(1):223-31.

6. Ramdasi S, Sarang S, Viswanathan C. Potential of mesenchymal stem cell based application in cancer. Int J Hematol Oncol Stem Cell Res Ther. 2015; 9(2):95.

7. da Silva ML, Chagastelles PC, Nardi NB. Mesenchymal stem cells reside in virtually all post-natal organs and tissues. J Cell Sci. 2006;119(11):2204-13.

8. Kuçi S, Kuçi Z, Schäfer R, Spohn G, Winter S, Schwab M, et al. Molecular signature of human bone marrow-derived mesenchymal stromal cell subsets. Sci Rep. 2019;9(1):1-10.

9. Lin W, Huang L, Li Y, Fang B, Li G, Chen L, et al. Mesenchymal stem cells and cancer: clinical challenges and opportunities. Biomed Res Int. 2019;2019:1-12.

10. Nombela-Arrieta C, Ritz J, Silberstein LE. The elusive nature and function of mesenchymal stem cells. Nat Rev Mol Cell Biol. 2011;12(2):126-31.

11. Dai L-J, Moniri MR, Zeng Z-R, Zhou JX, Rayat J, Warnock GL. Potential implications of mesenchymal stem cells in cancer therapy. Cancer Lett. 2011;305(1):8-20.

12. Lee MW, Ryu S, Kim DS, Lee JW, Sung KW, Koo HH, et al. Mesenchymal stem cells in suppression or progression of hematologic malignancy: current status and challenges. Leukemia. 2019;33(3):597-611.

13. Hmadcha A, Martin-Montalvo A, Gauthier BR, Soria B, Capilla-Gonzalez VJFiB, Biotechnology Therapeutic Potential of Mesenchymal Stem Cells for Cancer Therapy. Front Bioeng Biotechnol. 2020;8:43.

14. Bentzon JF, Stenderup K, Hansen F, Schroder H, Abdallah B, Jensen ta, et al. Tissue distribution and engraftment of human mesenchymal stem cells immortalized by human telomerase reverse transcriptase gene. Biochem Biophys Res Commun. 2005:330(3):633-640.

15. Huang $P$, Lin LM, Wu XY, Tang QL, Feng XY, Lin GY, et al. Differentiation of human umbilical cord Wharton's jelly-derived mesenchymal stem cells into germ-like cells in vitro. J Cell Biochem. 2010;109(4):747-54.

16. Sibov $T T$, Severino $P$, Marti L, Pavon L, Oliveira D, Tobo $P$, et al. Mesenchymal stem cells from umbilical cord blood: parameters for isolation, characterization and adipogenic differentiation. Cytotechnology. 2012;64(5): 511-21.

17. Stewart MC, Stewart AA. Mesenchymal stem cells: characteristics, sources, and mechanisms of action. Vet Clin. 2011;27(2):243-61.

18. Millán-Rivero JE, Nadal-Nicolás FM, García-Bernal D, Sobrado-Calvo P, Blanquer M, Moraleda JM, et al. Human Wharton's jelly mesenchymal stem cells protect axotomized rat retinal ganglion cells via secretion of antiinflammatory and neurotrophic factors. Sci Rep. 2018;8(1):1-14.

19. Ribeiro J, Pereira T, Amorim I, Caseiro AR, Lopes MA, Lima J, et al. Cell therapy with human MSCs isolated from the umbilical cord Wharton jelly associated to a PVA membrane in the treatment of chronic skin wounds. Int J Med Sci. 2014;11(10):979.

20. Hass RKC, Bohm S, Jacobs R. Different populations and sources of human mesenchymal stem cells (MSC): a comparison of adult and neonatal tissuederived MSC. Cell Commun Signal. 2011;9:12

21. Thilakavathy K, Nordin N, Ramasamy R, Ghoraishizadeh P, IMR R, Singh G. Characteristics of full-term amniotic fluid-derived mesenchymal stem cells in different culture media. Mesenchymal Stem Cells. 2017;39:2820853.

22. Xue Z, Wu X, Chen X, Liu Y, Wang X, Wu K, et al. Mesenchymal stem cells promote epithelial to mesenchymal transition and metastasis in gastric cancer though paracrine cues and close physical contact. J Cell Biochem. 2015;116(4):618-27. 
23. Gholizadeh-Ghaleh Aziz S, Fardyazar Z, Pashaiasl M. The human amniotic fluid mesenchymal stem cells therapy on, SKOV3, ovarian cancer cell line. Mol Genet Genomic Med. 2019;7(7):e00726.

24. Li L, Wang D, Zhou J, Cheng Y, Liang T, Zhang G. Characteristics of human amniotic fluid mesenchymal stem cells and their tropism to human ovarian cancer. Plos one. 2015;10(4):e0123350.

25. Lee HY, Hong IS. Double-edged sword of mesenchymal stem cells: cancerpromoting versus therapeutic potential. Cancer Sci. 2017;108(10):1939-46.

26. Underwood MA, Gilbert WM, Sherman MP. Amniotic fluid: not just fetal urine anymore. J Perinatol. 2005;25(5):341-8.

27. Loukogeorgakis SPC. Paolo concise review: amniotic fluid stem cells: the known, the unknown, and potential regenerative medicine applications. Stem Cells. 2017;35(7):1663-73.

28. Babaei G, Gholizadeh Ghaleh Aziz S, Alipour S, Naderi R. Comparison of traditional prenatal diagnosis procedures and cell-free DNA in maternal plasma as a new molecular approach for prenatal diagnosis. Int J Res Appl Basic Med Sci 2018:4(2):108-118.

29. Kang N, Hwang K, Kim S, Kim Y, Hyun S, Jeung E, et al. Potential antitumor therapeutic strategies of human amniotic membrane and amniotic fluidderived stem cells. Cancer Gene Ther. 2012;19(8):517-22.

30. Eslaminejad MB, Jahangir S. Amniotic fluid stem cells and their application in cell-based tissue regeneration. Int J Fertil Steril. 2012;6(3):147.

31. Atala A, Lanza R, Mikos T, Nerem R. Principles of regenerative medicine. Cambridge: Academic press; 2018.

32. Moschidou D, Mukherjee S, Blundell MP, Drews K, Jones GN, Abdulrazzak H, et al. Valproic acid confers functional pluripotency to human amniotic fluid stem cells in a transgene-free approach. Mol Ther. 2012;20(10):1953-67.

33. You Q, Tong X, Guan Y, Zhang D, Huang M, Zhang Y, et al. The biological characteristics of human third trimester amniotic fluid stem cells. J Int Med Res. 2009;37(1):105-12

34. Nadri S, Soleimani M. Comparative analysis of mesenchymal stromal cells from murine bone marrow and amniotic fluid. Cytotherapy. 2007;9(8):729-37.

35. Gholipourmalekabadi M, Farhadihosseinabadi B, Faraji M, Nourani MRJB. How preparation and preservation procedures affect the properties of amniotic membrane? How safe are the procedures? 2019.

36. Danieli P, Malpasso G, Ciuffreda MC, Cervio E, Calvillo L, Copes F, et al. Conditioned medium from human amniotic mesenchymal stromal cells limits infarct size and enhances angiogenesis. Stem Cells Transl Med. 2015 4(5):448-58

37. Zhang K, Cai Z, Li Y, Shu J, Pan L, Wan F, et al. Utilization of human amniotic mesenchymal cells as feeder layers to sustain propagation of human embryonic stem cells in the undifferentiated state. Cell Reprogram. 2011;13(4):281-8.

38. Tropel P, Platet N, Platel JC, Noël D, Albrieux M, Benabid AL, et al. Functional neuronal differentiation of bone marrow-derived mesenchymal stem cells. Stem Cells. 2006;24(12):2868-76.

39. Magatti M, De Munari S, Vertua E, Nassauto C, Albertini A, Wengler GS, et al. Amniotic mesenchymal tissue cells inhibit dendritic cell differentiation of peripheral blood and amnion resident monocytes. Cell Transplant. 2009; 18(8):899-914

40. Li J-Y, Ren K-K, Zhang W-J, Xiao L, Wu H-Y, Liu Q-Y, et al. Human amniotic mesenchymal stem cells and their paracrine factors promote wound healing by inhibiting heat stress-induced skin cell apoptosis and enhancing their proliferation through activating PI3K/AKT signaling pathway. Stem Cell Res Ther. 2019:10(1):1-17.

41. Silini AR, Cargnoni A, Magatti M, Pianta S, Parolini O. The long path of human placenta, and its derivatives, in regenerative medicine. Front Bioeng Biotechnol. 2015:3:162.

42. Steigman SA, Ahmed A, Shanti RM, Tuan RS, Valim C, Fauza DO. Sternal repair with bone grafts engineered from amniotic mesenchymal stem cells. J Pediatr Surg. 2009:44(6):1120-6.

43. Spitzhorn L-S, Rahman MS, Schwindt L, Ho H-T, Wruck W, Bohndorf M, et al. Isolation and molecular characterization of amniotic fluid-derived mesenchymal stem cells obtained from caesarean sections. Stem Cells Int. 2017;2017:5932706.

44. Wei X, Sun G, Zhao X, Wu Q, Chen L, Xu Y, et al. Human amnion mesenchymal stem cells attenuate atherosclerosis by modulating macrophage function to reduce immune response. Int J Mol Med. 2019:44(4):1425-35.

45. Sun B, Roh K-H, Park J-R, Lee S-R, Park S-B, Jung J-W, et al. Therapeutic potential of mesenchymal stromal cells in a mouse breast cancer metastasis model. Cytotherapy. 2009;11(3):289-98.
46. Qiao L, Xu Z, Zhao T, Zhao Z, Shi M, Zhao RC, et al. Suppression of tumorigenesis by human mesenchymal stem cells in a hepatoma model. Cell Res. 2008;18(4):500-7.

47. Jiao H, Guan F, Yang B, Li J, Song L, Hu X, et al. Human amniotic membrane derived-mesenchymal stem cells induce C6 glioma apoptosis in vivo through the BCl-2/caspase pathways. Mol Biol Rep. 2012;39(1):467-73.

48. Philipp D, Suhr L, Wahlers T, Choi Y-H, Paunel-Görgülü A. Preconditioning of bone marrow-derived mesenchymal stem cells highly strengthens their potential to promote IL-6-dependent M2b polarization. Stem Cell Res Ther. 2018;9(1):1-17.

49. Javan MR, Khosrojerdi A, Moazzeni SM. New insights into implementation of mesenchymal stem cells in cancer therapy: prospects for anti-angiogenesis treatment. Front Oncol. 2019:9:840.

50. Magatti M, Caruso M, De Munari S, Vertua E, De D, Manuelpillai U, et al. Human amniotic membrane-derived mesenchymal and epithelial cells exert different effects on monocyte-derived dendritic cell differentiation and function. Cell Transplant. 2015;24(9):1733-52.

51. Bauer D, Wasmuth S, Hennig M, Baehler H, Steuhl K-P, Heiligenhaus A. Amniotic membrane transplantation induces apoptosis in T lymphocytes in murine corneas with experimental herpetic stromal keratitis. Invest Ophthalmol Visual Sci. 2009:50(7):3188-98.

52. Kang N-H, Yi B-R, Lim SY, Hwang K-A, Baek YS, Kang K-S, et al. Human amniotic membrane-derived epithelial stem cells display anticancer activity in BALB/C female nude mice bearing disseminated breast cancer xenografts. Int J Oncol. 2012;40(6):2022-8.

53. Jablonski EM, Mattocks MA, Sokolov E, Koniaris LG, Hughes FM Jr, Fausto $\mathrm{N}$, et al. Decreased aquaporin expression leads to increased resistance to apoptosis in hepatocellular carcinoma. Cancer Lett. 2007; 250(1):36-46

54. Niknejad H, Yazdanpanah G, Ahmadiani A. Induction of apoptosis, stimulation of cell-cycle arrest and inhibition of angiogenesis make human amnion-derived cells promising sources for cell therapy of cancer. Cell Tissue Res. 2016;363(3):599-608

55. Jiang YJ, Kim P, Lu YF, Feingold KR. PPARgamma activators stimulate aquaporin 3 expression in keratinocytes/epidermis. Exp Dermatol. 2011; 20(7):595-9.

56. Bauer D, Hennig M, Wasmuth S, Baehler H, Busch M, Steuhl K-P, et al. Amniotic membrane induces peroxisome proliferator-activated receptor- $\gamma$ positive alternatively activated macrophages. Invest Ophthalmol Vis Sci. 2012;53(2):799-810.

57. You Q, Yao Y, Zhang Y, Fu S, Du M, Zhang G. Effect of targeted ovarian cancer therapy using amniotic fluid mesenchymal stem cells transfected with enhanced green fluorescent protein-human interleukin-2 in vivo. Mol Med Rep. 2015;12(4):4859-66.

58. Masiero M, Simões FC, Han HD, Snell C, Peterkin T, Bridges E, et al. A core human primary tumor angiogenesis signature identifies the endothelial orphan receptor ELTD1 as a key regulator of angiogenesis. Cancer Cell Int 2013;24(2):229-41

59. Lee J-K, Park S-R, Jung B-K, Jeon Y-K, Lee Y-S, Kim M-K, et al. Exosomes derived from mesenchymal stem cells suppress angiogenesis by downregulating VEGF expression in breast cancer cells. PloS one. 2013;8(12):39-52.

60. Otsu K, Das S, Houser SD, Quadri SK, Bhattacharya S, Bhattacharya J. Concentration-dependent inhibition of angiogenesis by mesenchymal stem cells. Blood. 2009;113(18):4197-205.

61. Niknejad H, Paeini-Vayghan G, Tehrani F, Khayat-Khoei M, Peirovi H. Side dependent effects of the human amnion on angiogenesis. Placenta. 2013; 34(4):340-5

62. Niknejad H, Yazdanpanah G. Opposing effect of amniotic membrane on angiogenesis originating from amniotic epithelial cells. J Med Hypotheses Ideas. 2014;8(1):39-41.

63. Deng L, Chen N, Li Y, Zheng H, Lei Q. CXCR6/CXCL16 functions as a regulator in metastasis and progression of cancer. Biochim Biophys Acta Rev Cancer. 2010;1806(1):42-9.

64. Feng $B$, Chen L. Review of mesenchymal stem cells and tumors: executioner or coconspirator? Cancer Biother Radiopharmaceuticals. 2009;24(6):717-21.

65. Kim JS, Kurie JM, Ahn Y-H. BMP4 depletion by miR-200 inhibits tumorigenesis and metastasis of lung adenocarcinoma cells. Mol Cancer. 2015;14(1):173.

66. Meng M-Y, Li L, Wang W-J, Liu F-F, Song J, Yang S-L, et al. Assessment of tumor promoting effects of amniotic and umbilical cord 
mesenchymal stem cells in vitro and in vivo. J Cancer Res Clin Oncol. 2019;145(5):1133-46.

67. Azoitei N, Diepold K, Brunner C, Rouhi A, Genze F, Becher A, et al. HSP90 supports tumor growth and angiogenesis through PRKD2 protein stabilization. Cancer Res. 2014;74(23):7125-36.

68. Jafari A, Rezaei-Tavirani M, Farhadihosseinabadi B, Taranejoo S, Zali H. HSP90 and co-chaperones: impact on tumor progression and prospects for molecular-targeted cancer therapy. Cancer invest. 2020;38(5):310-28.

69. Wang Q, Xia N, Li T, Xu Y, Zou Y, Zuo Y, et al. SUMO-specific protease 1 promotes prostate cancer progression and metastasis. Oncogene. 2013; 32(19):2493-8.

70. Niknejad H, Yazdanpanah G, Mirmasoumi M, Abolghasemi H, Peirovi H, Ahmadiani A. Inhibition of HSP90 could be possible mechanism for anti-cancer property of amniotic membrane. Med Hypotheses. 2013; 81(5):862-5.

71. Libby P. Inflammatory mechanisms: the molecular basis of inflammation and disease. Nutr Rev. 2007;65(suppl_3):S140-S6.

72. Mantovani A. Inflammation by remote control. Nat Rev Cancer. 2005; 435(7043):752-3.

73. Greten FR, Grivennikov SI. Inflammation and cancer: triggers, mechanisms, and consequences. Immunity. 2019;51(1):27-41.

74. Domínguez-López A, Bautista-de Lucio VM, Serafín-López J, Robles-Sánchez $\mathrm{E}$, Garfias Y. Amniotic membrane modulates innate immune response inhibiting PRRs expression and NF-KB nuclear translocation on limbal myofibroblasts. Exp Eye Res. 2014;127:215-23.

75. Singh N, Baby D, Rajguru JP, Patil PB, Thakkannavar SS, Pujari VB. Inflammation and cancer. Ann Afr Med. 2019:18(3):121.

76. Navas A, Magaña-Guerrero FS, Domínguez-López A, Chávez-García C, Partido G, Graue-Hernández EO, et al. Anti-inflammatory and antifibrotic effects of human amniotic membrane mesenchymal stem cells and their potential in corneal repair. Stem Cells Transl Med. 2018;7(12):906-17.

77. Magatti M, Vertua E, Cargnoni A, Silini A, Parolini O. The immunomodulatory properties of amniotic cells: the two sides of the coin. Cell Transplant. 2018; 27(1):31-44.

78. Li J, Koike-Soko C, Sugimoto J, Yoshida T, Okabe M, Nikaido T. Human amnion-derived stem cells have immunosuppressive properties on NK cells and monocytes. Cell Transplant. 2015;24(10):2065-76.

79. Pianta S, Signoroni PB, Muradore I, Rodrigues MF, Rossi D, Silini A, et al. Amniotic membrane mesenchymal cells-derived factors skew $T$ cell polarization toward Treg and downregulate Th1 and Th17 cells subsets. Stem Cell Rev Rep. 2015;11(3):394-407.

80. Onishi R, Ohnishi S, Higashi R, Watari M, Yamahara K, Okubo N, et al. Human amnion-derived mesenchymal stem cell transplantation ameliorates dextran sulfate sodium-induced severe colitis in rats. Cell Transplant. 2015;24(12): 2601-14.

81. Kang JW, Koo HC, Hwang SY, Kang SK, Ra JC, Lee MH, et al. Immunomodulatory effects of human amniotic membrane-derived mesenchymal stem cells. J Vet Sci. 2012;13(1):23-31.

82. Parekkadan B, Milwid JM. Mesenchymal stem cells as therapeutics. Annu Rev Biomed Eng. 2010;12:87-117.

83. Bitsika V, Roubelakis MG, Zagoura D, Trohatou O, Makridakis M, Pappa KI, et al. Human amniotic fluid-derived mesenchymal stem cells as therapeutic vehicles: a novel approach for the treatment of bladder cancer. Stem Cells Dev. 2012;21(7):1097-111.

84. Zhou J, Liang T, Wang D, Li L, Cheng Y, Guo Q, et al. IFNa-expressing amniotic fluid-derived mesenchymal stem cells migrate to and suppress HeLa cellderived tumors in a mouse model. Stem Cells Int. 2018;2018:1241323.

85. Cousin B, Ravet E, Poglio S, De Toni F, Bertuzzi M, Lulka H, et al. Adult stromal cells derived from human adipose tissue provoke pancreatic cancer cell death both in vitro and in vivo. Plos one. 2009:4(7):e6278.

86. Bruno S, Collino F, lavello A, Camussi G. Effects of mesenchymal stromal cell-derived extracellular vesicles on tumor growth. Front Immunol. 2014;5:382.

87. Magatti M, De Munari S, Vertua E, Parolini O. Amniotic membrane-derived cells inhibit proliferation of cancer cell lines by inducing cell cycle arrest. J Cell Mol Med. 2012;16(9):2208-18.

88. Riedel R, Pérez-Pérez A, Carmona-Fernández A, Jaime M, Casale R, Dueñas $\mathrm{JL}$, et al. Human amniotic membrane conditioned medium inhibits proliferation and modulates related microRNAs expression in hepatocarcinoma cells. Sci Rep. 2019;9(1):1-20.
89. Malumbres M, Barbacid M. Cell cycle, CDKs and cancer: a changing paradigm. Nat Rev Cancer. 2009;9(3):153-66.

90. Rhee K-J, Lee JI, Eom YW. Mesenchymal stem cell-mediated effects of tumor support or suppression. Int J Mol Sci. 2015;16(12):30015-33.

91. König J, Huppertz B, Desoye G, Parolini O, Fröhlich JD, Weiss G, et al. Amnion-derived mesenchymal stromal cells show angiogenic properties but resist differentiation into mature endothelial cells. Stem Cells Dev. 2012; 21(8):1309-20.

92. Zhao C, Wang $X$, Zhao Y, Li Z, Lin S, Wei Y, et al. A novel xenograft model in zebrafish for high-resolution investigating dynamics of neovascularization in tumors. Plos One. 2011:6(7):e21768.

93. Rani S, Ryan AE, Griffin MD, Ritter T. Mesenchymal stem cell-derived extracellular vesicles: toward cell-free therapeutic applications. Mol Ther. 2015;23(5):812-23.

94. Yang X, Hou J, Han Z, Wang Y, Hao C, Wei L, et al. One cell, multiple roles: contribution of mesenchymal stem cells to tumor development in tumor microenvironment. Cell Biosci. 2013;3(1):5.

95. Verseijden F, Posthumus-van Sluijs SJ, Pavljasevic P, Hofer SO, van Osch GJ, Farrell E. Adult human bone marrow-and adipose tissue-derived stromal cells support the formation of prevascular-like structures from endothelial cells in vitro. Tissue Eng A. 2010;16(1):101-14.

96. Hwang JH, Shim SS, Seok OS, Lee HY, Woo SK, Kim BH, et al. Comparison of cytokine expression in mesenchymal stem cells from human placenta, cord blood, and bone marrow. J Korean Med Sci. 2009;24(4):547-54.

97. Ong CT, Khoo YT, Tan EK, Mukhopadhyay A, Do D, Han HC, et al. Epithelialmesenchymal interactions in keloid pathogenesis modulate vascular endothelial growth factor expression and secretion. J Pathol. 2007;211(1): 95-108.

98. Khan S, Villalobos MA, Choron RL, Chang S, Brown SA, Carpenter JP, et al. Fibroblast growth factor and vascular endothelial growth factor play a critical role in endotheliogenesis from human adipose-derived stem cells. J Vasc Surg. 2017;65(5):1483-92.

99. Xiao S, Huang G, Wei Z, Nie K, Liu Z, Deng C, et al. IL-10 gene-modified human amniotic mesenchymal stem cells augment regenerative wound healing by multiple synergistic effects. Stem Cells Int. 2019;2019:9158016.

100. Quintero-Fabián S, Arreola R, Becerril-Villanueva E, Torres-Romero JC, AranaArgáez VE, Lara-Riegos J, et al. Role of matrix metalloproteinases in angiogenesis and cancer. Front Oncol. 2019:9:1370.

101. Zhang C, Yu L, Liu S, Wang YJPo. Human amnion-derived mesenchymal stem cells promote osteogenic and angiogenic differentiation of human adipose-derived stem cells. Plos One. 2017;12(10):e0186253.

102. Wu Q, Fang T, Lang H, Chen M, Shi P, Pang X, et al. Comparison of the proliferation, migration and angiogenic properties of human amniotic epithelial and mesenchymal stem cells and their effects on endothelial cells. Int J Mol Med. 2017;39(4):918-26.

103. Kocherova I, Bryja A, Mozdziak P, Angelova Volponi A, DyszkiewiczKonwińska M, Piotrowska-Kempisty $\mathrm{H}$, et al. Human umbilical vein endothelial cells (HUVECS) co culture with osteogenic cells: from molecular communication to engineering prevascularised bone grafts. Journal of clinical medicine. 2019;8(10):1602.

104. Jeong IS, Park Y, Ryu HA, An HS, Han JH, Kim S-W. Dual chemotactic factorssecreting human amniotic mesenchymal stem cells via TALEN-mediated gene editing enhanced angiogenesis. Int J Cardiol. 2018;260:156-62.

105. Salomon C, Ryan J, Sobrevia L, Kobayashi M, Ashman K, Mitchell M, et al. Exosomal signaling during hypoxia mediates microvascular endothelial cell migration and vasculogenesis. Plos One. 2013;8(7):e68451.

106. Gatti S, Bruno S, Deregibus MC, Sordi A, Cantaluppi V, Tetta C, et al. Microvesicles derived from human adult mesenchymal stem cells protect against ischaemia-reperfusion-induced acute and chronic kidney injury. Nephrol Dial Transplant. 2011;26(5):1474-83.

107. Bruno S, Grange C, Deregibus MC, Calogero RA, Saviozzi S, Collino F, et al. Mesenchymal stem cell-derived microvesicles protect against acute tubular injury. J Am Soc Nephrol. 2009;20(5):1053-67.

108. Dang LT, Lawson ND, Fish JE. MicroRNA control of vascular endothelial growth factor signaling output during vascular development. Arterioscler Thromb Vasc Biol. 2013;33(2):193-200.

109. Sedrakyan S, Villani V, Da Sacco S, Tripuraneni N, Porta S, Achena A, et al. Amniotic fluid stem cell-derived vesicles protect from VEGF-induced endothelial damage. Sci Rep. 2017;7(1):1-12.

110. Kato Y, Ozawa S, Miyamoto C, Maehata Y, Suzuki A, Maeda T, et al. Acidic extracellular microenvironment and cancer. Cancer Cell Int. 2013;13(1):89. 
111. Reiser-Erkan C, Erkan M, Pan Z, Bekasi S, Giese NA, Streit S, et al. Hypoxiainducible proto-oncogene Pim-1 is a prognostic marker in pancreatic ductal adenocarcinoma. Cancer Biol Ther. 2008;7(9):1352-9.

112. Zhang B, Wang M, Gong A, Zhang X, Wu X, Zhu Y, et al. HucMSC-exosome mediated-Wnt4 signaling is required for cutaneous wound healing. Stem Cells. 2015;33(7):2158-68

113. Rahmatizadeh F, Aziz SG-G, Khodadadi K, Ataei ML, Ebrahimie E, Rad JS, et al. Bidirectional and opposite effects of naïve mesenchymal stem cells on tumor growth and progression. Adv Pharm Bull. 2019;9(4):539.

114. Fallah A, Sadeghinia A, Kahroba H, Samadi A, Heidari HR, Bradaran B, et al. Therapeutic targeting of angiogenesis molecular pathways in angiogenesisdependent diseases. Biomed Pharmacother. 2019;110:775-85.

115. Xu G, Zhang Y, Zhang L, Ren G, Shi Y. The role of IL-6 in inhibition of lymphocyte apoptosis by mesenchymal stem cells. Biochem Biophys Res Commun. 2007:361(3):745-50.

116. Arno Al, Amini-Nik S, Blit PH, Al-Shehab M, Belo C, Herer E, et al. Human Wharton's jelly mesenchymal stem cells promote skin wound healing through paracrine signaling. Stem Cell Res Ther. 2014;5(1):28.

117. Harman RM, He MK, Zhang S, Van de Walle GR. Plasminogen activator inhibitor-1 and tenascin- $C$ secreted by equine mesenchymal stromal cells stimulate dermal fibroblast migration in vitro and contribute to wound healing in vivo. Cytotherapy. 2018;20(8):1061-76.

118. Ontsuka K, Kotobuki Y, Shiraishi H, Serada S, Ohta S, Tanemura A, et al. Periostin, a matricellular protein, accelerates cutaneous wound repair by activating dermal fibroblasts. Exp Dermatol. 2012;21(5):331-6.

119. Castilho RM, Squarize CH, Gutkind JS. Exploiting PI 3 K/m TOR signaling to accelerate epithelial wound healing. Oral Dis. 2013;19(6):551-8.

120. Cetinkaya B, Unek G, Kipmen-Korgun D, Koksoy S, Korgun ET. Effects of human placental amnion derived mesenchymal stem cells on proliferation and apoptosis mechanisms in chronic kidney disease in the rat. Int J Stem Cells. 2019;12(1):151.

121. Lao G, Ren M, Wang X, Zhang J, Huang Y, Liu D, et al. Human tissue inhibitor of metalloproteinases-1 improved wound healing in diabetes through its anti-apoptotic effect. Exp Dermatol. 2019;28(5):528-35

122. Zou X, Zhang G, Cheng Z, Yin D, Du T, Ju G, et al. Microvesicles derived from human Wharton's jelly mesenchymal stromal cells ameliorate renal ischemia-reperfusion injury in rats by suppressing CX3CL1. Stem Cell Res Ther. 2014;5(2):40.

123. Dong L, Pu Y, Zhang L, Qi Q, Xu L, Li W, et al. Human umbilical cord mesenchymal stem cell-derived extracellular vesicles promote lung adenocarcinoma growth by transferring miR-410. Cell Death Dis. 2018; 9(2):1-13.

124. Ruscetti M, Quach B, Dadashian EL, Mulholland DJ, Wu H. Tracking and functional characterization of epithelial-mesenchymal transition and mesenchymal tumor cells during prostate cancer metastasis. Cancer Res. 2015;75(13):2749-59.

125. Wei SC, Fattet L, Tsai JH, Guo Y, Pai VH, Majeski HE, et al. Matrix stiffness drives epithelial-mesenchymal transition and tumour metastasis through a TWIST1-G3BP2 mechanotransduction pathway. Nat Cell Biol. 2015;17(5):678-88

126. Son $\mathrm{H}$, Moon A. Epithelial-mesenchymal transition and cell invasion. Toxicol Res. 2010;26(4):245-52

127. Lamouille S, Xu J, Derynck R. Molecular mechanisms of epithelialmesenchymal transition. Nat Rev Mol Cell Biol. 2014;15(3):178

128. Chen J, Ji T, Wu D, Jiang S, Zhao J, Lin H, et al. Human mesenchymal stem cells promote tumor growth via MAPK pathway and metastasis by epithelial mesenchymal transition and integrin a5 in hepatocellular carcinoma. Cell Death Dis. 2019;10(6):1-12.

129. Tan J, Xu X, Tong Z, Yu Q, Lin Y, Kuang W. Decreased osteogenesis of adult mesenchymal stem cells by reactive oxygen species under cyclic stretch: a possible mechanism of age related osteoporosis. Bone Res. 2015;3:15003.

130. Takigawa H, Kitadai Y, Shinagawa K, Yuge R, Higashi Y, Tanaka S, et al. Mesenchymal stem cells induce epithelial to mesenchymal transition in colon cancer cells through direct cell-to-cell contact. Neoplasia. 2017; 19(5):429-38.

131. Plaks V, Kong N, Werb Z. The cancer stem cell niche: how essential is the niche in regulating stemness of tumor cells? Cell Stem Cell. 2015; 16(3):225-38.

132. Wu S, Wang Y, Yuan Z, Wang S, Du H, Liu X, et al. Human adiposederived mesenchymal stem cells promote breast cancer MCF7 cell epithelialmesenchymal transition by cross interacting with the TGF $3 / S$ mad and PI3KJAKT signaling pathways. Mol Med Rep. 2019;19(1):177-86.
133. Lange-Consiglio A, Perrini C, Tasquier R, Deregibus MC, Camussi G, Pascucci $L$, et al. Equine amniotic microvesicles and their anti-inflammatory potential in a tenocyte model in vitro. Stem Cells Dev. 2016;25(8):610-21.

134. Lange-Consiglio A, Lazzari B, Perrini C, Pizzi F, Stella A, Cremonesi F, et al. MicroRNAs of equine amniotic mesenchymal cell-derived microvesicles and their involvement in anti-inflammatory processes. Cell Transplant. 2018;27(1): $45-54$.

\section{Publisher's Note}

Springer Nature remains neutral with regard to jurisdictional claims in published maps and institutional affiliations. 